\title{
Traditional Chinese Medicine Baicalin Suppresses mESCs Proliferation through Inhibition of miR-294 Expression
}

\author{
Jian Wang Jacob Masika ${ }^{a, b, c}$ Jianxia Zhou ${ }^{a, b}$ Jun Wanga, ${ }^{a, b}$ Minjie Zhu ${ }^{a, b}$ \\ Hongyan Luo ${ }^{a, b}$ Xinwu Hu $u^{a, b}$ Liangpin Zhang ${ }^{a, b}$ Ming Tang ${ }^{a, b}$ Linlin Gao \\ Jürgen Heschelere Huamin Liang ${ }^{a, b}$
}

\begin{abstract}
aDepartment of Physiology, Chinese-German Stem Cell Center, the Key Laboratory of Drug Target Research and Pharmacodynamic Evaluation, Hubei Province; School of Basic Medicine, Huazhong University of Science and Technology, Wuhan, China, 'Institute of Brain Research, Huazhong University of Science and Technology, Wuhan, China; 'Department of Medical Physiology, Faculty of Health Sciences, Egerton University, Kenya; 'Department of Xiangyang Hospital, Hubei University of Medicine, Xiangyang, China; ${ }^{\mathrm{e}}$ Institute of Physiology, University of Cologne, Cologne, Germany
\end{abstract}

\section{Key Words}

MESC proliferation $•$ Baicalin $•$ MicroRNAs $\bullet$ C-jun $•$ C-fos

\begin{abstract}
Background: Traditional Chinese herbal medicines (TCMs) have been widely used against a broad spectrum of biological activities, including influencing the cardiac differentiation from mouse embryonic stem cells (mESCs). However, their effects and mechanisms of action on ESCs proliferation remain to be determined. The present study aimed to determine the effect of three TCMs, baicalin, ginsenoside Rg1, and puerarin, on mESCs proliferation and to elucidate the possible mechanism of their action. Methods: Cell proliferation was examined with a cell proliferation assay Cell Counting Kit-8 (CCK-8), propidium iodide (PI) staining was used to visualize cell cycle. The mRNA expression level of c-myc, c-fos, c-jun, GAPDH and microRNAs were measured by quantitative real time RT-PCR. Results: We found that baicalin $50 \mu \mathrm{M}$ suppressed the proliferation of mESCs as observations in more cells in G1 phase and less cells in either $S$ phase or G2/M phase. Moreover, baicalin suppressed the expressions of c-jun and c-fos in mESCs and down-regulated the expression of miR-294. Overexpression of miR-294 in mESCs significantly reversed the effects of baicalin both on $\mathrm{mESC}$ proliferation and c-fos/c-jun expression. Conclusions: Baicalin down-regulation of miR-294 may be its key mechanism of action in decreasing $\mathrm{mESC}$ s proliferation.
\end{abstract}

J. Wang and J. Masika equal contribution to this manuscript.

Huamin Liang

KARGER 125
Hangkong Road 13, Wuhan 430030 (China)

Tel. +862783692622, Fax +862783692608

E-Mail lianghuamin76@163.com 


\section{Introduction}

Embryonic stem cells (ESCs) are derived from the inner cell mass (ICM) of blastocysts. They are capable of unlimited self-renewal, as well as proliferation, and maintain pluripotency in vitro to differentiate into all three germ layers endoderm, mesoderm, and ectoderm, as the most primitive stem cells. Thus, ESCs have great potential applications in regenerative medicine and serve as model to study molecular embryogenesis [1]. Consequently, in recent decades ESCs have become one of the research hotspots in many fields of medical research [2].

The proliferation ability is one of the interests in ESCs research, which is mainly focused on the exogenous/endogenous transcription factors, signal pathway molecules, small molecular synthetic material, and gene regulations. The proliferation ability of ESCs is closely related to the regulation of ESC cell cycle, and ESCs are characterized with a short G1 period [3]. Some of the somatic cells like bone marrow, liver, and skin cells, have a greater regeneration and proliferation potential with short G1 period, while most of the other somatic cells regeneration potential is considered to be low with a long quiescent G0 phase and the proliferation ability is regarded as poor [3]. Cell cycle regulation has two main restriction point, G1/S restriction point and G2/M restriction point. It has been shown that microRNAs (miRNAs) can regulate cell cycle-related gene expression of transcription factors regulatory pathways and thus affect stem cell self-renewal, proliferation and differentiation, maintaining the balance of two biological processes [4]. These specific cell-cycle regulating miRNAs in ESCs are known as Embryonic Stem cell-specific Cell Cycle-regulating miRNAs (ESCC-miRNAs) [5], in which the miR-290 cluster in mESCs is highly profiled and plays an important role in the proliferation of mESCs [3].

Two types of genes, oncogenes and cancer suppressor genes, are related to cell proliferation. Activation of oncogenes leads to increased expression of genes that promote cell cycle progression and cell proliferation. Because some of them already exist in the cell, they are called proto-oncogene [6]. The proto-oncogenes, c-myc, c-fos, and c-jun, are early response proteins that regulates cell cycle genes. They can promote positive regulators of cell cycle such as Cyclin and Cdk binding [7] which usher the cell into the cell cycle and are usually considered as promoters of G1/S restriction point in the process. Whether c-jun and c-fos can act as detectors of cell proliferation genes and can play important roles in ESCs proliferation and differentiation remains unexplored.

In China, traditional Chinese medicines (TCMs) have been used for the treatment of various types of diseases for thousands of years. Moreover, some studies have revealed that the active components of TCMs have the ability to influence stem cell proliferation, differentiation and apoptosis [8-12]. These active components regulate either the transcription factors [13] or the cell cycle-related genes thereby affecting stem cell differentiation, proliferation and apoptosis. Our previous data have revealed that TCMs baicalin, puerarin and ginsenoside Rg1 influence the cardiac differentiation of mESCs [14-16] by changing the expression of the cardiac-specific transcriptional factors. Whether they could also exert effects on mESCs proliferation by modifying the expression of mESC-miRNAs remains to be determined. Thus we aimed to explore this possibility. The obtained data showed that baicalin suppressed the proliferation of ESCs by regulating the expression of proto-oncogenes c-fos and c-jun via miR-294 downregulation.

\section{Materials and Methods}

Cell Culture of mESCs

The mouse embryonic stem cell line D3 (D3-mESCs) was obtained from ATCC (Manassas, USA) and cultured using Dulbecco's modified Eagle's medium (DMEM) (Gibco, USA) supplemented with $15 \%$ (v/v) fetal bovine serum (FBS) (Gibco, USA), $2 \mathrm{mM}$ L-glutamine (Gibco/Invitrogen, USA), $50 \mathrm{mM}$ $\beta$-mercaptoethanol (Gibco/Invitrogen, USA), 1x non-essential amino acids (Gibco/Invitrogen, USA) and $100 \mathrm{IU} / \mathrm{ml}$ penicillin/streptomycin(Gibco/Invitrogen, USA) at $37{ }^{\circ} \mathrm{C}$ and $5 \% \mathrm{CO}_{2}$. ESCs were cultured on a 
feeder layer of mitomycin C-inactivated mouse embryonic fibroblasts (MEFs) in the presence of $1000 \mathrm{U} / \mathrm{ml}$ recombinant mouse leukemia inhibitory factor (Chemicon, Temecula, USA) to remain in an undifferentiated state.

Cell Proliferation Assays

Cell proliferation was examined $48 \mathrm{~h}$ after inoculation with a cell proliferation assay using Cell Counting Kit-8 (CCK-8) (DOJINDO, Japan) according to the manufacturer's instructions. Briefly, cells were seeded in 96-well plates at 1000 cells/well with $100 \mu \mathrm{l}$ 15\% DMEM, and after $48 \mathrm{~h}, 10 \mu \mathrm{l}$ CCK-8 solution/ well was added. The optical density was measured using the microplate reader at a wavelength of $450 \mathrm{~nm}$.

\section{Cell Cycle Assay}

Propidium iodide (PI) staining was used to visualize cell cycle. Approximately $1 \times 10^{6}$ cells were pelleted by centrifugation at 1500 r.p.m. for $5 \mathrm{~min}$, washed with $1 \mathrm{ml}$ cold Dulbecco's phosphate-buffered saline (PBS) without $\mathrm{MgCl}_{2}$ or $\mathrm{CaCl}_{2}$ thereafter processed for cell cycle analysis. Cells were resuspended in $4 \mathrm{ml}$ cold $70 \%$ ethanol at $4{ }^{\circ} \mathrm{C}$ for $1 \mathrm{~h}$, then centrifuged at 1500 r.p.m. for $5 \mathrm{~min}$. The pellet was washed with cold PBS, re-suspended in $500 \mu \mathrm{l} \mathrm{PBS}$, and incubated with $10 \mu \mathrm{l} 1 \mathrm{mg} / \mathrm{ml}$ RNase $(20 \mu \mathrm{g} / \mathrm{ml}$ final concentration) for $30 \mathrm{~min}$. The cells were incubated with propidium iodide $(50 \mathrm{mg} / \mathrm{ml})$ on ice for $1 \mathrm{~h}$ in the dark. The cell cycle distribution was analyzed using FACS Caliber instrument (BD Biosciences, San Jose, CA, USA). Nuclear debris and overlapping nuclei were gated out.

\section{RNA Isolation and Revere Transcription}

Total RNA including miRNAs was extracted from ESCs using TRIzol (Invitrogen, USA). Revere transcription was performed on 2 ng of total RNA. Briefly, 2 ng of total RNA was reverse transcribed to cDNA. First strand cDNA of proto-oncogenes was synthesized by using M-MLV reverse transcriptase (Invitrogen, USA) with dT (18) oligo, and the First strand cDNA of miRNAs was synthesized by using M-MLV reverse transcriptase (Invitrogen, USA) with specific stem loop miRNA RT Primer (Ribo, China).

\section{Real-time PCR}

For real-time quantitative RT-PCR of c-myc, c-fos, c-jun and GAPDH, the SYBR-Green PCR MasterMix (TOYOBO CO., LTD, Japan) was used with gene-specific primers listed in Table 1. Real-time quantitative PCR was performed using $1.6 \mu \mathrm{l}$ the first strand cDNA, $0.4 \mu \mathrm{l} 10 \mathrm{mM}$ forward and reverse primers, $10 \mu \mathrm{l}$ SYBRGreen PCR MasterMix to total $20 \mu$ volume. The PCR processing consisted of 40 cycles of $10 \mathrm{~s}$ denaturation at $95^{\circ} \mathrm{C}, 20 \mathrm{~s}$ annealing at $60^{\circ} \mathrm{C}$, and $15 \mathrm{~s}$ extension at $72^{\circ} \mathrm{C}$. All reactions were run in triplicate. For real-time quantitative PCR of miR-294, miR-295, miR-291a, miR-291b, miR-93 and U6, the SYBR-Green PCR MasterMix was used with stem loop miRNA primers (Ribo, China). Reaction and signal detection were measured by Mx3000P real-time PCR system (Agilent Stratagene, USA). The CT values of proto-oncogenes and miRNAs were converted into absolute copy numbers using a standard curve for GAPDH and U6 miRNA, respectively.

\section{Overexpression of miRNA-294}

A single-cell suspension of $2 \times 10^{5} \mathrm{mESC}$ was plated in the tissue culture dishes to achieve a $50 \sim 60 \%$ confluence for approximately $24 \mathrm{~h}$. Once the confluence was achieved, the adherent cells were transfected using Lipofectamine ${ }^{\mathrm{TM}} 2000$ (Invitrogen, USA), with mmu-miR-294 mimic (Ribo, China) or empty vector (control) in OPTI-MEM (Gibco, USA) according to the manufacturer's instruction. After 2 days of cell culture, the cells were trypsinized and collected for further experiments.

\section{Agents}

Baicalin, puerarin, and ginsenoside Rg1 were purchased from Nanjing Qingze Medical Technology Co., Ltd, dissolved in dimethyl sulfoxide (DMSO, Sigma Co.) to stock concentrations, and stored at -20 ${ }^{\circ} \mathrm{C}$ in the dark.

Table 1. Primers used for real time RT-PCR

\begin{tabular}{lll}
\hline Gene & & Primer sequence 5'-3' \\
\hline GAPDH & Forward & AACTTTGGCATTGTGGAAGG \\
& Reverse & GGATGCAGGGATGATGTTCT \\
c-myc & Forward & GCTCTCCATCCTATGTTGCGG \\
& Reverse & TCCAAGTAACTCGGTCATCATCT \\
c-jun & Forward & GAAACGACCTTCTACGACGA \\
& Reverse & TGAGAAGGTCCGAGTTCTTG \\
c-fos & Forward & GAATCCGAAGGGAACGGAATAAG \\
& Reverse & CAATCTCAGTCTGCAACGCA \\
\hline
\end{tabular}



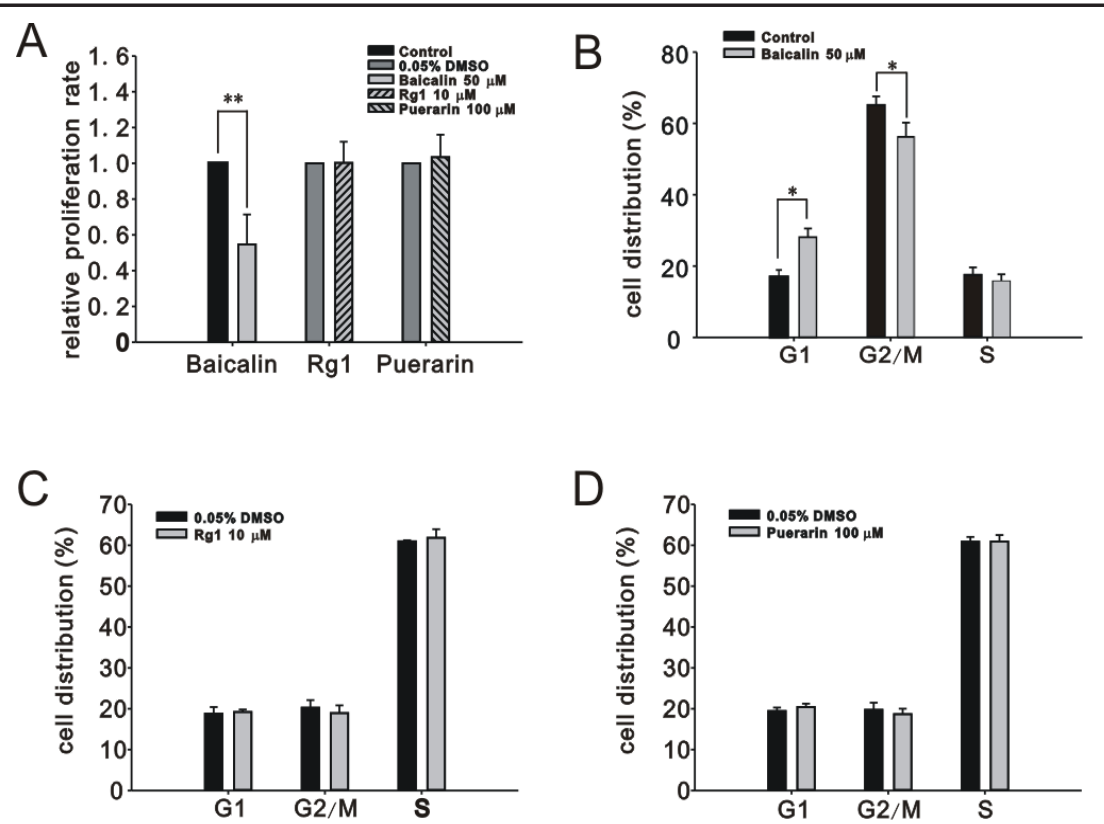

Fig. 1. Effects of three TCMs on the proliferation of mESCs (A) Baicalin $50 \mu \mathrm{M}$ inhibited the proliferation of mESCs to $0.55 \pm 0.04$ vs. control $(p<0.01, \mathrm{n}=14)$. Ginsenoside $\operatorname{Rg} 110 \mu \mathrm{M}$ and puerarin $100 \mu \mathrm{M}$ had no effect on the mESCs proliferation $(p>0.05, \mathrm{n}=10)$. (B) Baicalin accumulated more mESCs in G1 phases $(p<0.05$, $n=4)$ and less cells in S phase $(p<0.05, n=4)$. (C), (D) Ginsenoside Rg1 and puerarin did not change the cell distribution.

\section{Statistical Analysis}

Data were expressed as the mean \pm S.E.M. Comparisons between two groups were made by a twotailed Student's t test. A p value of $<0.05$ was considered to denote statistical significance.

\section{Results}

\section{Baicalin Suppressed mESCs Proliferation}

We first examined whether the three different TCMs, baicalin, ginsenoside Rg1, and puerarin, could influence the proliferation of mESCs. Dose dependent effects of each TCM were tested (data not shown) and the dose of maximum effects was applied in the following experiments. As shown in Fig. 1A, baicalin $50 \mu \mathrm{M}$ suppressed the proliferation of mESCs to $0.55 \pm 0.04$ of control $(p<0.01, \mathrm{n}=14)$, while ginsenoside Rg1 $10 \mu \mathrm{M}(1.00 \pm 0.03$ of control, $p>0.05, \mathrm{n}=10$ ) and puerarin $100 \mu \mathrm{M}$ had no significant effects on the proliferation of mESCs $(1.03 \pm 0.03$ of control, $p>0.05, \mathrm{n}=10$ ).

The cell cycle analysis on mESCs showed that most of mESCs distributed in $\mathrm{S}$ phase $(65.15 \pm 2.42 \%)$ and less mESCs in G1 $(17.31 \pm 1.82 \%)$ and G2/M phases $(17.54 \pm 2.09 \%)$. Baicalin $50 \mu \mathrm{M}$ accumulated more mESCs in G1 phase (up to $28.20 \pm 2.33 \%, p<0.05, \mathrm{n}=4$ ) and less cells in $\mathrm{S}$ phase (down to $56.25 \pm 3.95 \%, p<0.05, \mathrm{n}=4$ ) and $\mathrm{G} 2 / \mathrm{M}$ phase (down to $15.55 \pm 1.95 \%, p>0.05, \mathrm{n}=4)$ (Fig. 1B). Neither $\operatorname{Rg} 1(10 \mu \mathrm{M})$ nor puerarin $(100 \mu \mathrm{M})$ changed the cell distribution significantly (Fig. 1C-D).

The above observations in the three TCMs were paralleled well with the expression level of proto-oncogenes c-myc, c-jun and c-fos (Fig. 2). The expressions of c-jun and c-fos were suppressed by baicalin $50 \mu \mathrm{M}$ to $27.29 \pm 0.05 \%$ and $55.08 \pm 0.06 \%$ vs. control $(p<0.01, \mathrm{n}=5)$, respectively (Fig. 2A). In contrast, neither ginsenoside Rg1 $10 \mu \mathrm{M}$ (Fig. 2B) nor puerarin 100 $\mu \mathrm{M}$ (Fig. 2C) significantly influenced the expressions of the three proto-oncogenes $(p>0.05$ vs. $0.05 \%$ DMSO, $n=5$ ). Therefore these data suggested that in the three tested TCMs, baicalin 
Fig. 2. Effect of TCMs on the expression level of proto-oncogenes

(A) Baicalin $50 \mu \mathrm{M}$ downregulated c-jun and c-fos ( $\mathrm{p}<0.05, \mathrm{n}=5)$. (B), (C) Ginsenoside Rg1 and puerarin did not change the expression of the proto-oncogenes $(\mathrm{p}>0.05$, $\mathrm{n}=5$ ).

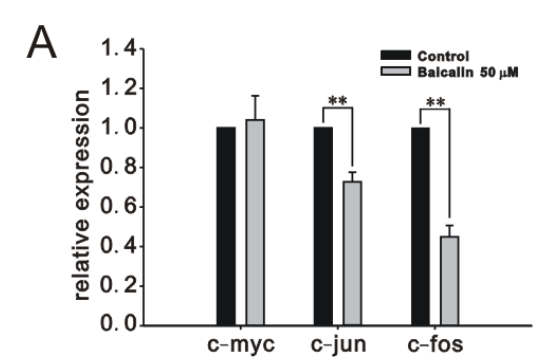

B

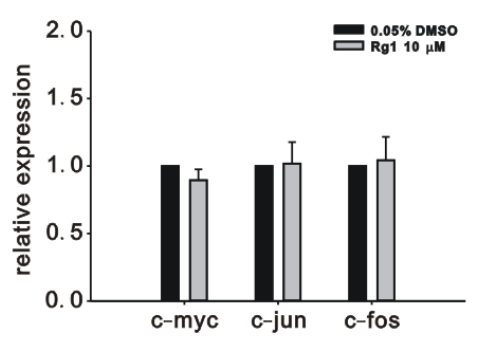

C

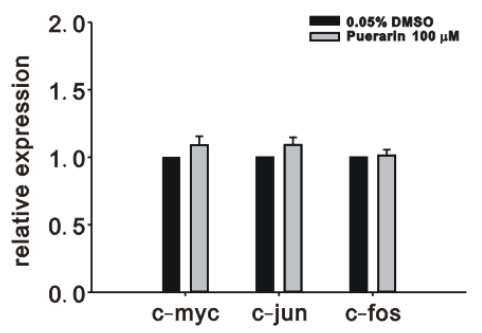

\section{A}

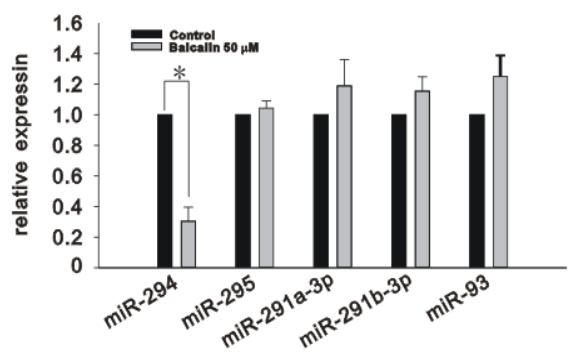

B

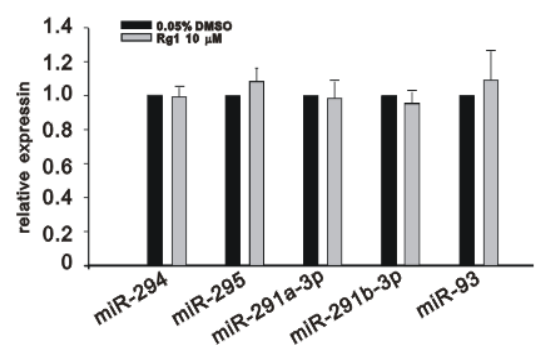

C

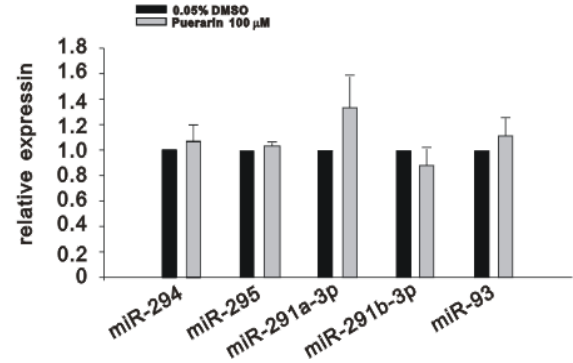

Fig. 3. Effect of TCMs on the expression level of members of miR-290 cluster (A) Effect of baicalin on miR290 cluster expression level. Baicalin $50 \mu \mathrm{M}$ downregulated the expression of miR-294 $(p<0.05, \mathrm{n}=4)$ while had no effect on the other members of miR-290 cluster ( $p>0.05, \mathrm{n}=4)$. (B) Effect of ginsenoside Rg1 $10 \mu \mathrm{M}$ on miR-290 cluster expression level. Rg1 $10 \mu \mathrm{M}(p>0.05, \mathrm{n}=4)$. (C) Effect of purerarin $100 \mu \mathrm{M}$ on miR-290 cluster expression level $(p>0.05, \mathrm{n}=4)$.

was the only one which significantly suppressed the mESC proliferation. Such suppression was accompanied with the G1 arrest, S stage suppression, and the down-regulation of protooncogenes c-jun and c-fos.

Baicalin downregulated miR-294 Expression in mESCs

MiR-290 cluster participates in regulating the proliferation of mESCs and some drugs might directly influence the expression level of miRNAs, thus we next investigated whether the TCMs affect the proliferation of mESCs by miR-290 cluster. The results showed that baicalin $50 \mu \mathrm{M}$ obviously downregulated the expression of miR-294 to 30.39 $\pm 9.21 \%$ compared with 


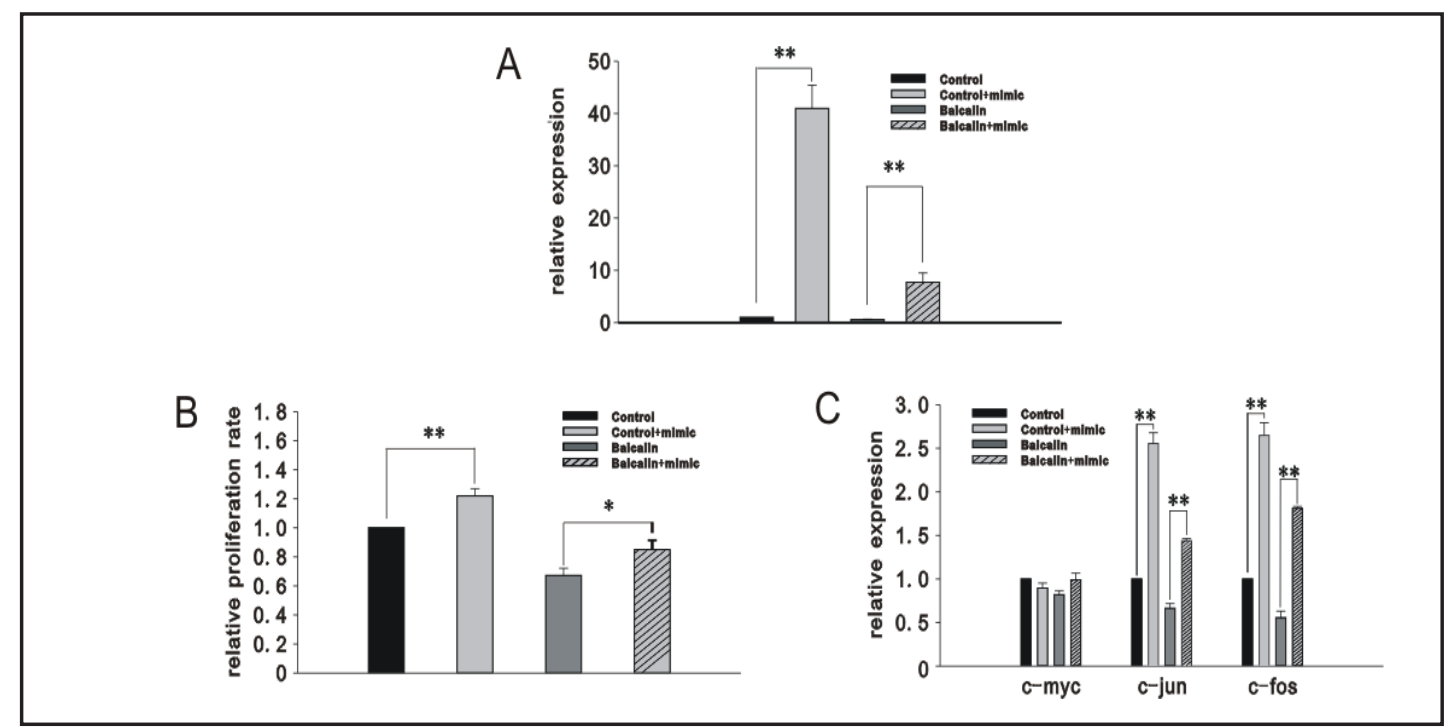

Fig. 4. Overexpression of miR-294 regulated the mESCs proliferation and the expression of protooncogenes. (A) With lipofectamin $2000^{\mathrm{TM}}$ transfection the miR-294 mimic to mESCs for $24 \mathrm{~h}$, the expression of miR-294 obviously was upregulated up to $40.97 \pm 4.44$ folds vs. vector control $(p<0.01, \mathrm{n}=4)$. The miR294 expression of in baicalin and miR-294 co-treated group was upregulated to $7.71 \pm 1.78$ folds vs. vector control $(p<0.01, \mathrm{n}=4)$. (B) Transfection the miR-294 mimic to mESCs elevated the proliferation rate $(p<0.01$, $\mathrm{n}=4)$, and rescued the proliferation rate of baicalin treated group $(p<0.05, \mathrm{n}=4)$. (C) Overexpression of miR294 upregulated the expression of c-jun and c-fos $(p<0.01, n=4)$, and rescued the downregulation of these genes induced by baicalin $(p<0.01, \mathrm{n}=4)$.

control $(p<0.05, \mathrm{n}=4)$ (Fig. 3A). Meanwhile, ginsenoside Rg1 $10 \mu \mathrm{M}$ and puerarin $100 \mu \mathrm{M}$ did not significantly alter the expressions of each member of miR-290 cluster $(p>0.05, n=4)$. (Fig. 3B-C). This strongly indicated that baicalin might inhibit mESC proliferation by downregulating miR-294.

Overexpression of miR-294 in mESCs Reversed the Alterations in the mESC Proliferation and the Expression of c-jun and c-fos Induced by Baicalin

To determine whether miR-294 is essential in baicalin-induced changes in mESCs, we transfected mESCs with miR-294 mimic by lipofectamin $2000^{\mathrm{TM}}$. With lipofectamin $2000^{\mathrm{TM}}$ transfection the miR-294 mimic to mESCs for $24 \mathrm{~h}$, the expression of miR-294 was upregulated to $40.97 \pm 4.44$ folds vs. vector control $(p<0.01, \mathrm{n}=4)$. The miR-294 expression in baicalin and miR-294 co-treated group was upregulated to $7.71 \pm 1.78$ folds vs. vector control $(p<0.01, \mathrm{n}=4)$, suggesting that baicalin indeed inhibited miR-294 expression and the miR-294 transfection rescued the downregulation of miR-294 by baicalin (Fig. 4A).

With overexpression of miR-294, the proliferation was elevated up to $1.22 \pm 0.05$ vs. vector control $(p<0.01, \mathrm{n}=4)$, as well as reversed the proliferation of baicalin treated group from $0.67 \pm 0.05$ to $0.85 \pm 0.06(p<0.05, n=4)$ (Fig. 4B). Moreover, the overexpression of miR294 upregulated the expression of $c$-jun and c-fos by $2.56 \pm 0.12$ and $2.65 \pm 0.14$ folds vs. vector control, respectively $(p<0.01, \mathrm{n}=4)$, and rescued the downregulation of these genes induced by baicalin from $0.66 \pm 0.05$ to $1.44 \pm 0.02$, and from $0.55 \pm 0.07$ to $1.81 \pm 0.02$ fold vs. vector control $(p<0.01, \mathrm{n}=4)$ (Fig. $4 \mathrm{C})$ for $\mathrm{c}$-jun and $\mathrm{c}$-fos respectively.

\section{Discussion}

Traditional Chinese medicines (TCMs) and their active ingredients have been reported to regulate the biological processes such as proliferation, differentiation and apoptosis 
by the regulation of gene expression of transcription factors and cytokines [7, 9]. In the present research, we investigated the possibility of three TCMs, baicalin, ginsenoside Rg1, and puerarin, to modify the expression of miRNAs that affect the proliferation of ESCs. The current study demonstrated that only baicalin significantly suppressed the mESC proliferation and was accompanied with the G1 arrest, S phase suppression, and the downregulation of proto-oncogenes c-jun and c-fos. Treatment of cells with this flavone resulted in downregulation of miR-294 expression in mESCs while overexpression of miR-294 in mESCs reversed the alterations in the mESC proliferation and the expression of c-jun and c-fos induced by baicalin.

Baicalin has been used for the treatment of inflammation, cardiovascular disease and microbial infections [7]. Its antitumor activity has also been reported in a variety of human cancer cell lines [8-10]. This activity is demonstrated by the baicalin induced cell cycle arrest and growth suppression [8]. Similarly, baicalin significantly increases the fraction of G0-G1 phase cells, but decreases the numbers of G2-M and S phase cells in vascular smooth muscle cells (VSMCs). Inhibition of cell proliferation by baicalin is due to its ability to reduce proliferating cell nuclear antigen (PCNA) expression and to elevate p27 levels in a concentration-dependent manner, coinciding with cell cycle arrest [12]. The present study showed that baicalin inhibited ESCs proliferation. The findings that baicalin inhibited ESC proliferation with increase in G1-phase cells and decrease in S phase cells were in good agreement with the observations in cancer cells and VSMCs.

The proto-oncogenes, c-myc, c-fos, and c-jun, can influence positive regulators of cell cycle such as Cyclin and Cdk binding [7]. c-myc together with other factors can induce somatic cells to become pluripotent stem cells and its abnormal expression in somatic cells is often associated with the occurrence and development of tumors [17]. c-jun and c-fos are often associated with c-myc in the development of cancer and may be used as indicators of cell proliferation as they alter the expression levels of factors and genes directly associated with cell growth [7]. Whether c-jun and c-fos can act as detectors of cell proliferation genes as well as play important roles in ESCs proliferation is unclear. The present study, however, showed that baicalin $50 \mu \mathrm{M}$ significantly reduced c-jun and c-fos, which might account for the results of ESCs proliferation analysis and the cell cycle analysis.

Self-renewal capacity and proliferation in ESCs are maintained by a shortening of their cell cycle that leads to a rapid cell division $[18,19]$. miR-290 cluster plays an important role in the proliferation of mouse embryonic stem cells (mESCs) by regulating the cell cycle [20]. The present data showed that miR-294 was suppressed by baicalin. Overexpression of miR-294 reversed the alterations in the mESC proliferation and the expression of c-jun and c-fos induced by baicalin. MiR-294 may regulate p21 protein (Cdc42/Rac)-activated kinase 7 (Pak7) and mitogen-activated protein kinase 8 (MAKP8) expression. Pak7 plays an important role in the process of signal transduction as a class of protein factors [3]. MAKP8, also called JNK1, can bind with the downstream signaling elements of the c-jun (junD, c-jun, ATF-Smad4, p53) to promote cell proliferation [3]. Although c-jun and c-fos are not the direct gene targets of miR-294, they may associate with JNK signal transduction pathways to regulate the downstream targets therefore participate in the regulation of proliferation of ESCs. In the present study, baicalin downregulated miR-294, thereby regulating c-jun and c-fos expression and inhibition of ESCs proliferation. This mechanism may also propose a new perspective of baicalin activity in cancer therapy. Further research is warranted to firmly establish the present study findings and explore more opportunities for the use of baicalin as a research tool and as a therapeutic agent in the treatment of diseases. The present study was limited to murine embryonic stem cells which may reduce its generalizability to human applications because substantial differences between animal models and humans exist (21). However, the findings of this work adds a piece of knowledge to the understanding of baicalin action and therefore further studies with human embryonic stem cells or human induced pluripotent stems cells are needed to extend the findings of the present study.

In summary, baicalin significantly suppressed mESC proliferation which was accompanied with G1 arrest, S phase suppression and the down-regulation of proto- 
oncogenes c-jun and c-fos via inhibition of miR-294. MiRNAs influence stem cell self-renewal and these miRNAs are tightly regulated in complex molecular networks. Understanding the extent and function of these networks in development and the influence of pharmacological agents such as baicalin will greatly enhance our knowledge of both developmental and disease states and treatment of diseases.

\section{Acknowledgments}

This work is supported by a grant from National Nature Science Foundation of China (No.: 81100818).

\section{Disclosure Statement}

All the authors have no conflict of interest.

\section{References}

1 Wobus AM, Boheler KR: Embryonic stem cells: prospects for developmental biology and cell therapy. Physiol Rev 2005;852:635-678.

2 Hescheler J, Fleischmann BK, Lentini S, Maltsev VA, Rohwedel J, Wobus AM, Addicks: Embryonic stem cells: a model to study structural and functional properties in cardiomyogenesis. Cardiovasc Res 1997;36:149162.

- 3 Wang Y, Melton C, Li YP, Shenoy A, Zhang XX, Subramanyam D, Blelloch R: miR-294/miR-302 promotes proliferation, suppresses G1-S restriction point, and inhibits ESC differentiation through separable mechanisms. Cell Rep 2013; 4:99-109.

4 Melton C, Judson RL, Blelloch R: Opposing microRNA families regulate self-renewal in mouse embryonic stem cells. Nature 2010;463:621-626.

5 Wang Y, Medvid R, Melton C, Jaenisch R, Blelloch R: DGCR8 is essential for microRNA biogenesis and silencing of embryonic stem cell self-renewal. Nat Genet 2007;39:380-385.

6 Neganova I, Zhang X, Atkinson S, Lako M. Expression and functional analysis of G1 to S regulatory components reveals an important role for CDK2 in cell cycle regulation in human embryonic stem cells. Oncogene. 2009;28:20-30.

7 Lu Y, Joerger R, Wu C. Study of the chemical composition and antimicrobial activities of ethanolic extracts from roots of Scutellaria baicalensis Georgi. J Agric Food Chem 2011;59:10934-10942.

-8 Chao JI, Su WC, Liu HF. Baicalein induces cancer cell death and proliferation retardation by the inhibition of CDC2 kinase and survivin associated with opposite role of p38 mitogen-activated protein kinase and AKT. Mol Cancer Ther 2007;6:3039-3048.

-9 Ma Z, Otsuyama K, Liu S, Abroun S, Ishikawa H, Tsuyama N, Obata M, Li FJ, Zheng X, Maki Y, Miyamoto K, Kawano MM: Baicalein, a component of Scutellaria radix from Huang-Lian-Jie-Du-Tang (HLJDT), leads to suppression of proliferation and induction of apoptosis in human myeloma cells. Blood 2005;105:33123318.

10 Lee HZ, Leung HW, Lai MY, Wu CH. Baicalein induced cell cycle arrest and apoptosis in human lung squamous carcinoma CH27 cells. Anticancer Res 2005;25:959-964.

11 Woo AY, Cheng CH, Waye MM: Baicalein protects rat cardiomyocytes from hypoxia/reoxygenation damage via a prooxidant mechanism. Cardiovasc Res 2005;65:244-253.

12 Dong LH, Wen JK, Miao SB, Jia Z, Hu HJ, Sun RH, Wu Y, Han M: Baicalin inhibits PDGF-BB-stimulated vascular smooth muscle cell proliferation through suppressing PDGFR $\beta$-ERK signaling and increase in p27 accumulation and prevents injury-induced neointimal hyperplasia. Cell Res 2010;20:1252-1262.

13 Wu J, Pan Z, Cheng M, Shen Y, Yu H, Wang Q, Lou Y: Ginsenoside Rg1 facilitates neural differentiation of mouse embryonic stem cells via GR-dependent signaling pathway. Neurochem Int 2013;62:92-102. 


\section{Cellular Physiology Cell Physiol Biochem 2015;35:1868-1876

and Biochemistry

14 Tang M, Yin M, Tang M, Liang H, Yu C, Hu X, Luo H, Baudis B, Haustein M, Khalil M, Sarić T, Hescheler J, Xi J: Baicalin maintains late-stage functional cardiomyocytes in embryoid bodies derived from murine embryonic stem cells. Cell Physiol Biochem 2013;32:86-99.

15 Cheng Y, Wang L, Tang M, Yin M, Cui Y, Liang H, Song Y, Hu X, Luo H, Gao Y, Wang J, Hescheler J, Xi J: Effects of puerarin on cardiac differentiation and ventricular specialization of murine embryonic stem cells. Cell Physiol Biochem 2013;32:789-800.

16 Wang L, Cui Y, Tang M, Hu X, Luo H, Hescheler J, Xi J: Puerarin facilitates T-tubule development of murine embryonic stem cell-derived cardiomyocytes. Cell Physiol Biochem 2014;34:383-392.

17 Yuen MF, Wu PC, Lai VC, Lau JY, Lai CL. Expression of c-Myc, c-Fos, and c-jun in hepatocellular carcinoma. Cancer 2001;91:106-112.

18 Orford KW, Scadden DT: Deconstructing stem cell self-renewal: genetic insights into cell-cycle regulation. Nat Rev Genet 2008;9:115-128.

19 Stead E, White J, Faast R, Conn S, Goldstone S, Rathjen J, Dhingra U, Rathjen P, Walker D, Dalton S: Pluripotent cell division cycles are driven by ectopic $\mathrm{Cdk} 2$, cyclin $\mathrm{A} / \mathrm{E}$ and E2F activities. Oncogene 2002;21:8320-8333.

20 Wang Y, Baskerville S, Shenoy A, Babiarz JE, Baehner L, Blelloch R. Embryonic stem cell-specific microRNAs regulate the G1-S transition and promote rapid proliferation. Nat Genet 2008;40:1478-1483.

21 Post MJ, Laham R, Sellke FW, Simons M. Therapeutic angiogenesis in cardiology using protein formulations. Cardiovasc Res 2001;49:522-531. 